\title{
24-HOUR FETAL/MATERNAL MONITORING SYSTEM BASED ON PHONOCARDIOGRAM ANALYSIS
}

\author{
Dmitry Zhdanov ${ }^{\mathrm{a} 1,2}$, Artem Bureev ${ }^{1,2}$, Yana Kosteley ${ }^{1,2}$ \\ ${ }^{1}$ Limited Liability Company Diagnostica+, 634055, Tomsk, Russia \\ ${ }^{2}$ National Research Tomsk State University, 634050, Tomsk, Russia,
}

\begin{abstract}
The article describes an engineering prototype of the Device for 24-hour monitoring over the functional state of the fetus and mother's cardiovascular system in the antenatal period of pregnancy. The Device is a hardware and software system that ensures the registration and analysis of fetus and mother's phonocardiograms with further processing and interpretation. The interpretation process is designed to timely detect conditions that constitute a threat to a fetus. The Device operates as part of a telemedicine network that allows obstetricians-gynecologists managing pregnant women to remotely control their state. The article describes the Device design concept and features of its specific components. The engineering prototype of the Device was used to carry out biomedical tests that confirmed its suitability for 24-hour monitoring over the functional state of fetus and mother's cardiovascular system in home settings.
\end{abstract}

\section{Introduction}

Long-term control over the state of fetus in the $2^{\text {nd }}$ and $3^{\text {rd }}$ trimesters of pregnancy has been relevant for more than 100 years [1]. Despite all medical, scientific and technical achievements, the problem of maintaining fetus' life and health in normal conditions and in case of various pregnancy pathologies has not been solved completely yet [2-4]. The analysis of the most recent data (2016) shows that more than a half of the intrauterine fetal death (IUFD) episodes take place in home settings [2, 5].

The share of antenatal mortality in the stillbirth rate reaches $77.0 \%$. Very many of these cases happen at the late stages of pregnancy when emergency delivery is indicated. About $90 \%$ of the antenatal death episodes happen in home settings or rural areas where it is hard to receive emergency medical care. At the same time, antenatal death is preceded by longterm intrauterine fetal agony. The primary causes of fetal death at the late stages of pregnancy have been determined, and the most important causes are timely undiagnosed intrauterine hypoxia and acute gestation course disorders $[2,6]$.

The experts from the World Health Organization consider 24-hour monitoring over pregnant woman's state of health and fetus development to be the most effective method of

\footnotetext{
a Corresponding author: D_S_Zhdanov@mail.ru
} 
reducing the antenatal mortality rate [2,7]. However, now the state of fetus is objectively controlled only episodically by means of cardiotocography (CTG). At the same time, there are several problems connected with the use of CTG. The most serious issues are the following [2,7]:

- ultrasound signals capture not only the cardiac structures of a fetus, but also large blood vessels. The amplitude of Doppler signals reflected from them is sometimes not lower than the amplitude of signals reflected from cardiac structures, and it is impossible to distinguish between these signals via simple methods;

- $\quad$ exposure to energy is undesirable for a developing fetus: there are still no unambiguous data on its impact on a fetus;

- the prognostic value of CTG does not exceed 10-14 days, which is absolutely insufficient in case of the actual threat of antenatal fetal death and requires regular updates of test findings;

- CTG is performed at clinical laboratories of functional diagnostics, which can be inaccessible for many pregnant women and requires hospitalization.

An alternative solution is to detect parameters similar to the CTG indicators on the basis of fetal electrocardiograms (FECG). However, singling out low-frequency $10-30 \mathrm{mcV}$ FECG signals against the background of external noise and interference, pregnant woman's movements and respiratory activity is a quite sophisticated problem. Digital signal processing methods require considerable computation capacities [8]. Therefore registering FECG and building CTG on its basis are usually performed at clinical laboratories, which is often challenging.

Previously, the authors provided an analytical review of the contemporary state of this problem and described the existing approaches and methods that allow assessing the state of fetus in a mother's womb. This review can be found in [9].

To solve the problem of 24-hour maternal/fetal monitoring, in 2014-2016, the development team of LLC Diagnostica+, including the authors of this article, decided to develop a hardware and software system that would ensure the continuous monitoring of the cardiovascular system (CVS) of both the pregnant woman and her fetus in the antenatal period of gestation. At the same time, the system under development had to be suitable for use outside medical institutions (in home settings), had to have a feature of remote monitoring over physician's examinations and test findings and be able to detect conditions constituting a threat to a fetus. It would allow providing timely medical care to save a fetus and maintain mother's health.

\section{Materials and Methods}

As a result, the development team created an engineering prototype of the Device that ensures 24-hour monitoring over the functional state of a fetus and mother's cardiovascular system. The Device is a hardware and software system that registers fetal and maternal phonocardiograms (PCG) from the surface of a pregnant woman's body and sends them to specialized software. The Device software allows performing the preliminary processing of phonocardiograms and using them to build fetal and maternal cardiointervalogramms (CIG). These cardiointervalogramms are analyzed to assess the fetal and maternal CVS and detect conditions constituting a threat to a fetus.

The Device hardware operates in the following way (Figure 1). The acoustic sensors located in the mother's abdominal and cardiac areas register the acoustic signals of fetal and maternal heart beats and convert them into electric signals. These signals are transferred to an analog unit where they are amplified and filtered to reduce the impact of interferences and unwanted noises on the device. 
Then a microcontroller (microcontroller unit) uses an analog-to-digital converter to transform analog signals into a digital code. These data are saved in a built-in memory and shared with a software component by means of a wireless data transfer unit (Bluetooth).

The control and indication elements are used to display the status and operation mode of the prototype Device hardware, notify of dangerous conditions and operate the hardware component of the Device.

The power unit provides power supply within a necessary voltage range and currents of the component blocks, as input voltage changes due to the discharge of the battery. The charge level control unit is used to monitor the voltage level of the battery.

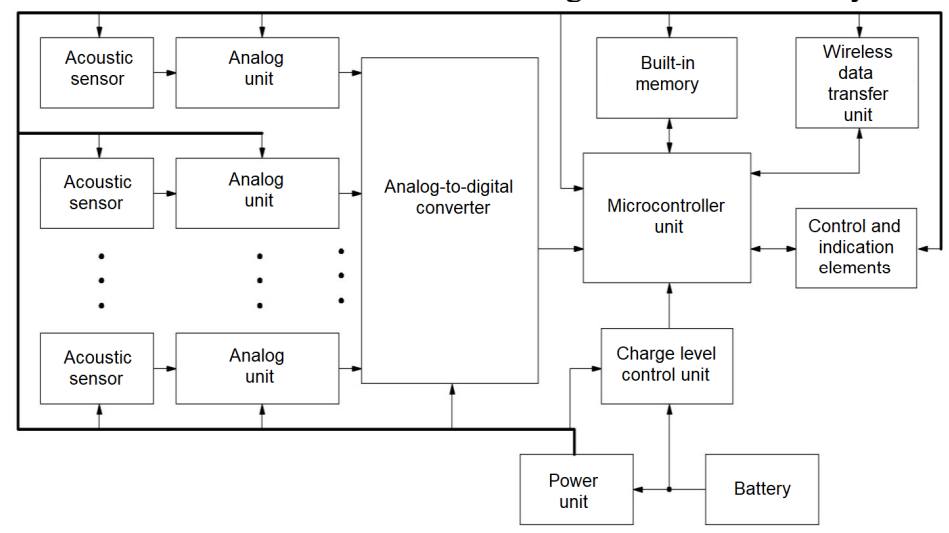

Fig. 1. Functional scheme of the Device hardware.

The specially developed piezoceramic sensors put into a soundproof body are used as acoustic sensors. These sensors allow achieving an average signal/noise ratio of 23.5-35 dB depending on the quality of contact between the sensor and pregnant woman's skin.

The Device software consists of several interconnected components responsible for different tasks:

1) Mobile application (Patient MWS) installed into a pregnant woman's smartphone. It is designed to receive PCGs from the Device hardware, perform preliminary processing (remove the sounds of rubbing against the patient's skin, detect dangerous conditions) and transfer PCGs and analysis findings to a remote server (Application Server). If conditions constituting a threat to a fetus are detected, the application sends commands to the prototype Device hardware informing it of a need for sound and visual signaling and sends alarm messages (in addition to independent sound and visual signaling) to the specialist who manages this patient and her relatives informing them of a need to provide emergency medical care;

2) Server software (Application Server). It is meant to receive, store and provide (upon the request from Physician AWS) patient data and test findings;

3) Software performing the functions of an obstetrician-gynecologist automated work station (Physician AWS). It is meant to keep electronic patient records, attach specific samples of the prototype Device hardware components to them, add (enter to the balance sheet) new samples of hardware components and review test findings on the functional state of the fetal and maternal CVS in both delayed and real-time modes. In addition, this software can send alarm messages via internal mail distribution;

4) Mobile application (Physician MWS) installed into the smartphone of the obstetrician-gynecologist who manages this patient. This is a mobile version of the Physician AWS that allows the obstetrician-gynecologist to review the findings of ongoing or previous tests and check internal mail regardless whether they are at their work place now or not. 


\section{Results and Discussion}

The applicability of the prototype Device (Figure 2) was assessed in 2016 during biomedical trials involving both the development team and experienced obstetriciansgynecologists. The trials were carried out in strict compliance with the developed biomedical trial program. 50 patients took part in these trials. The patients were informed of the goal of these trials and action principle of the prototype Device and gave their informed consent. The results received from the Device were compared with reference equipment, i.e. the UNICOS-01 and MAK-2Ch fetal monitors.

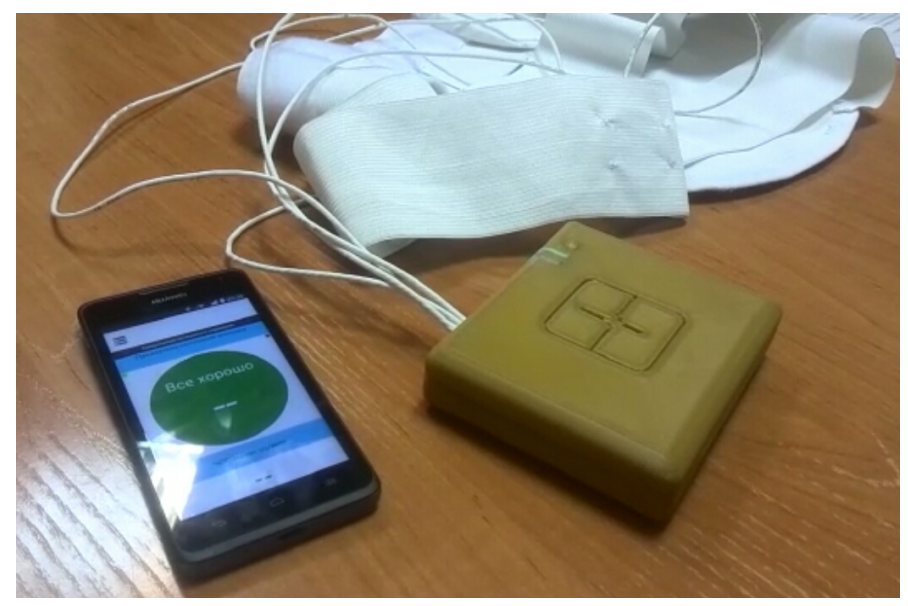

Fig. 2. External design of the prototype Device.

Right before the trial, the obstetricians-gynecologists used the Physician AWS interface to register the electronic records of the patients who took part in the trial and to attach the Device hardware sample to them. Then the sensors of reference equipment and the Device hardware component were attached to the surface of pregnant women's body, and the process of registering data on the fetal CVS was launched under constant surveillance by the obstetrician-gynecologist (Figure 3).

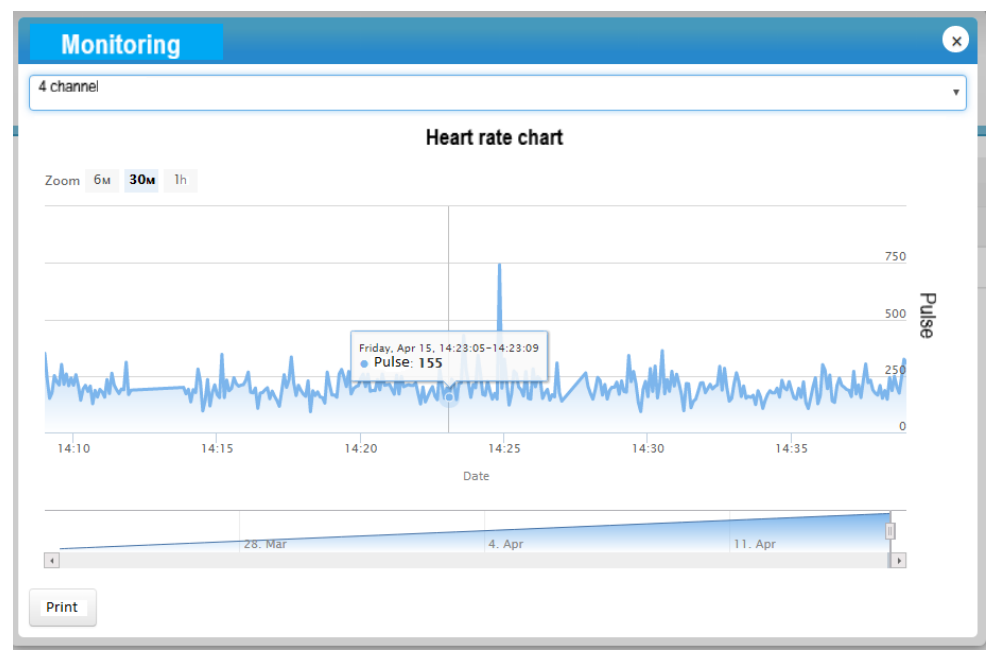

Fig. 3. Physician AWS interface during remote monitoring. 
No episodes of fetal state deterioration were detected during the trials. The PCG analysis findings obtained via the prototype Device were characterized by a margin of error amounting to $\pm 2 \mathrm{BPMs}$ vs. the reference devices. Overall, the trials confirmed the applicability of the Device for 24-hour fetal/maternal monitoring.

\section{Conclusion}

The work deliverables show that phonocardiography allows assessing the state of the fetal CVS with an accuracy rate comparable to that of the commonly used CTG method. At the same time, the accuracy of CIG construction is characterized by the margin of error amounting to \pm 2 BPMs. Figure 4 demonstrates an example of a CIG created as the result of processing a fetal PCG registered on the pregnant women's abdominal surface.

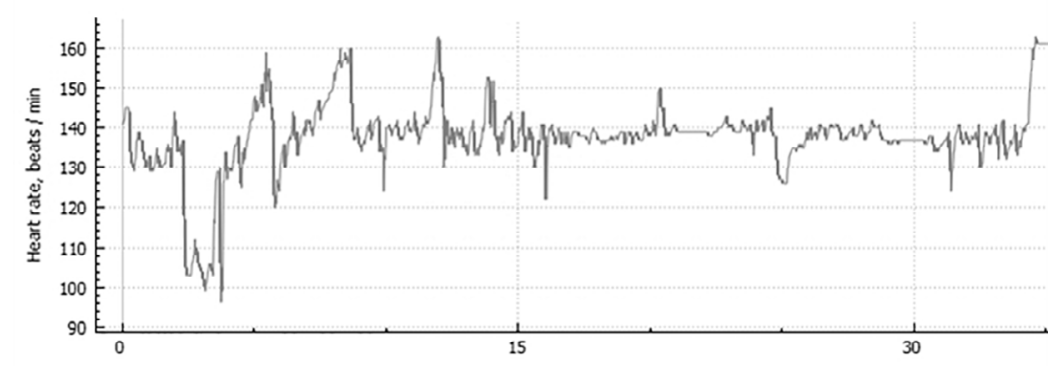

Fig. 4. Example of a CIG created by the Device on the basis of fetal PCG analysis findings.

Using the method of registering and analyzing fetal and maternal phonocardiograms allows assessing the state of the fetal CVS not only at medical institutions but also at any other place, as this method requires no special skills and additional equipment and means.

\section{Conflicts of Interests}

The authors declare that there is no conflict of interest regarding the publication of this paper.

These findings have been obtained within the framework of implementing Agreement No.14.579.21.0019 (unique project identifier - RFMEFI57914X0019) on the subject "Developing a device for 24-hour fetal and maternal health monitoring during pregnancy via control over cardiovascular parameters based on acoustic data" concluded between LLC Diagnostica+ and the Ministry of Education and Science of the Russian Federation.

\section{References}

[1] J. W. Ballantyne, Brit. Med. J. 3222, 583 (1922) doi: https://doi.org/10.1016/S00029378(25)90967-2

[2] P.R. Melinte, G.S. Dragoi, P. Paitici, I. Dinca, E. Patrascu1, Rom. J. Leg. Med. 23, 69 (2015) doi: 10.4323/rjlm.2015.69

[3] R.A. Pilliod, J.M. Page, R.M. Burwick, A.J. Kaimal, Y.W. Cheng, A.B. Caughey, Am. J. Obstet. Gynecol. 213, 410.e1 (2015) doi: 10.1016/j.ajog.2015.05.022

[4] A.P. South, K.M. Stutey, J. Meinzen-Derr, Am. J. Obstet. Gynecol. 209, 114.e1 (2013) doi: 10.1016/j.ajog.2013.04.032 
[5] A.S. McLennan, C. Gyamfi-Bannerman, C.V. Ananth, J.D. Wright, Z. Siddiq, M.E. D'Alton, F.M. Friedman, Am. J. Obstet. Gynecol. 217, 80.e1 (2017) doi: 10.1016/j.ajog.2017.03.002

[6] J. Adam, Rev. Obstet. Gynecol 5, e132 (2012)

[7] Levels \& Trends In Child Mortality (United Nations Children's Fund, New York, 2015)

[8] R.M. Rangayyan, Biomedical Signal Analysis (Wiley-IEEE Press, New-York, 2015)

[9] A.Sh. Bureev, D.S. Zhdanov, N.N. Zilberman, E.Yu. Kiseleva, S.Yu. Yuriev, Biosci. Biotech. Res. Asia 12, 1743 (2015) 